\title{
Repeat Expansion Disorders: Mechanisms and Therapeutics
}

\author{
Lisa M. Ellerby ${ }^{1}$ (I)
}

Published online: 6 January 2020

(C) The American Society for Experimental NeuroTherapeutics, Inc. 2020

\section{Introduction}

Repeat expansion disorders are a class of genetic diseases that are caused by expansions in DNA repeats. The DNA repeats come in various sizes from single nucleotides to dodecamers or longer. The threshold at which the repeat expansions become symptomatic varies with the specific disease. There are over 40 distinct diseases now known to be caused by these expansions in DNA sequence. Remarkable progress during the last three decades has defined causative mutations that drive pathogenesis in a large number of these diseases. Expansion of CAG, GCG, CTG, CGG, and CAAA repeats both in coding and non-coding sequences in distinct genes results in a diverse group of diseases with mechanisms linked to protein levels or toxicity, RNA, and/or both (Fig. 1).

As a polyglutamine (polyQ) expansion disease researcher, I was drawn to the field two decades ago when Drs. Hayden, Bredesen, and Wellington and I discovered that the majority of disease-causing polyQ proteins were substrates for caspases and that this pathway has a critical role in the mechanisms of neuronal cell death observed in patients [1-9]. Most importantly the community of patients who have diseases caused by expansions of DNA repeats and their families continue to inspire us to move forward with new discoveries and therapeutics treatments.

In this issue of Neurotherapeutics, an international group of experts review the molecular mechanisms and therapeutics for many of these diseases. Highlights from the reviews are summarized below and presented in order of the discovery of the causative mutation underlying these diseases (see Fig. 1).

Invited Review Molecular Pathogenesis and Advances in Therapeutics of Repeat Expansion

Disorders, guest edited by Dr. Lisa M. Ellerby

Lisa M. Ellerby

lellerby@buckinstitute.org

1 Buck Institute for Research on Aging, 8001 Redwood Blvd, Novato, CA 94945, USA
Spinal and Bulbar Muscular Atrophy/Kennedy's Disease In 1991, the cause of Kennedy's disease was shown to be a CAG expansion in the androgen receptor $(A R)$ gene [10]. Spinal and bulbar muscular atrophy (SBMA) is a slow progressive neuromuscular disorder in which the lower motor neurons and muscles degenerate. SBMA is X-linked and therefore mainly affecting males, with some exceptions discussed in the Arnold and Merry review [11]. The symptoms include gynecomastia, testicular atrophy, and reduced fertility, all which correlate with the known function of AR as a transcription factor binding androgen hormones. AR is involved in the male reproductive and skeletal system and female fertility. Given the known function of AR, the normal lifespan of SBMA patients, and the rapid progress in the field, it is surprising that after almost thirty years of investigation, we do not have a therapeutic treatment for this disease. Merry reviews the complexity of the underlying pathogenesis of this disorder and highlights steps that may lead to therapeutics for SBMA/Kennedy's disease [11].

Huntington's Disease In 1993, the cause of Huntington's disease was found to be a CAG expansion in exon 1 of the huntingtin gene (HTT) [12]. The disease protein contains a polyglutamine expansion in the $\mathrm{N}$-terminal region of the Huntingtin protein (HTT). Among the polyQ diseases, HD is the best studied. Several key questions in the field that continue to provide clues for therapeutic approaches in the field are [1] what are the cell-autonomous and non-cell autonomous mechanisms that are triggered by the mutant protein?; [2] what are the key molecular mechanisms that drive the disease and how can we appropriately model the human condition?; [3] what is the basis of preferentially vulnerability of distinct neuronal populations?; and finally, how does DNA repair mechanism impact HD and other DNA repeat expansions diseases? Maiuri et al. describe the emerging importance of DNA repair mechanisms in HD and other repeat expansion disorders [13]. Creus-Muncunill and Ehrlich review the detailed and elegant studies directed at understanding the cellautonomous and non-autonomous pathogenic mechanisms in HD [14]. Naphade et al. describe the rapid progress using 


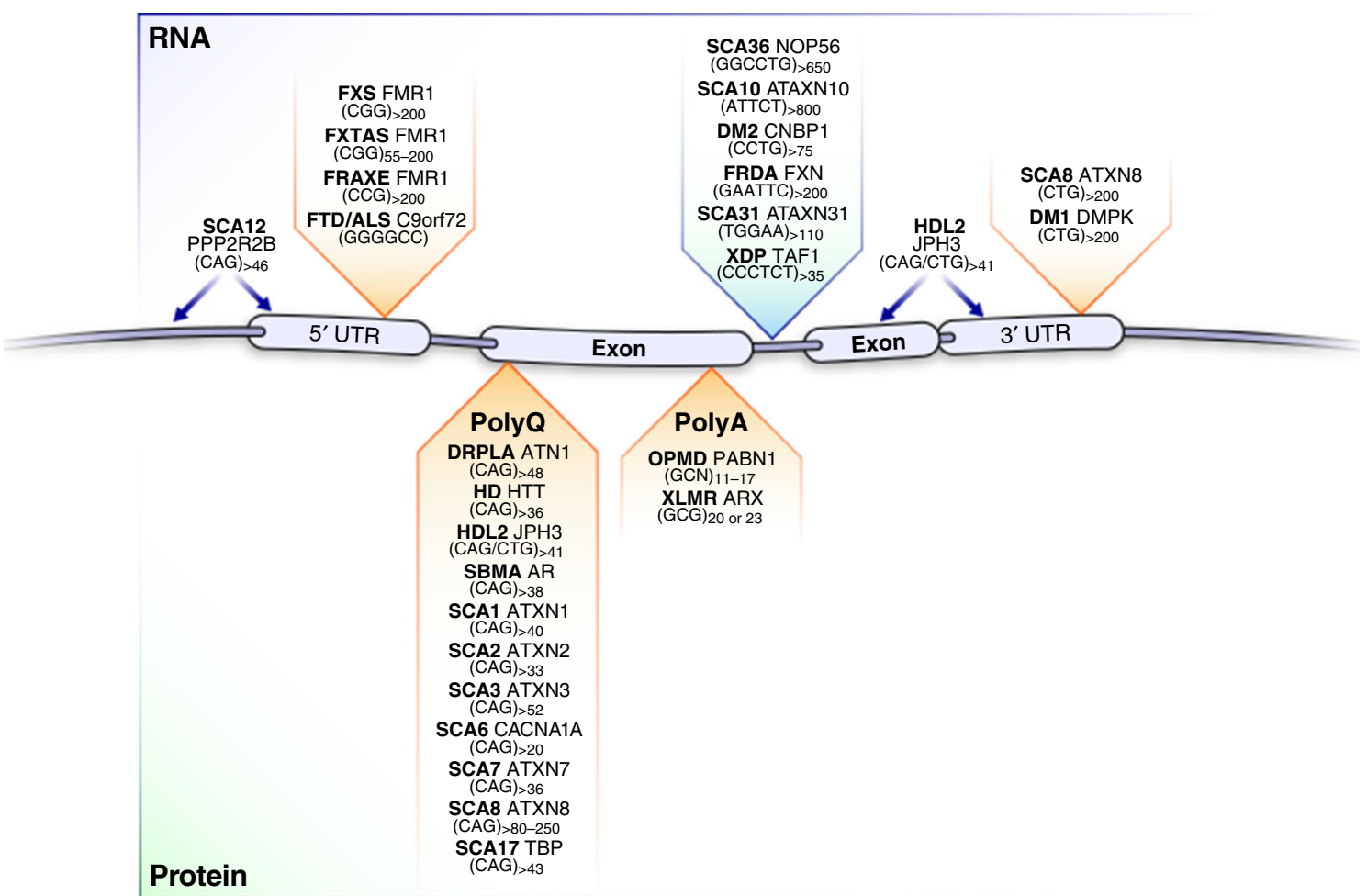

Fig. 1 The repeat expansion disorders, a class of genetic diseases that are caused by expansions DNA repeats. The location of the expansion occurs in exonic and intronic regions of the gene

human patient-induced pluripotent stem cells to model HD and other polyQ diseases [15].

SCA1 The genetic cause of Spinocerebellar ataxia type 1 (SCA1) was reported in 1993 [16]. SCA1 is an autosomaldominant disorder characterized by neurodegeneration of the cerebellum, spinal cord, and brainstem and has a polyQ expansion in the ataxin-1 protein. Srinivasan and Shakkottai review the advances in understanding SCA1 disease biology and the challenges in the field to developing tailored therapeutics for the distinct SCAs [17].

SCA3 Spinocerebellar ataxia type 3 (SCA3), also known as Machado-Joseph disease (MJD), is caused by a polyQ expansion in the ataxin-3 protein [18]. Ataxin-3 is a ubiquitin ligase and Da Silva et al. present a unifying molecular mechanism for disease pathogenesis focusing on the disruption of protein homeostasis [19]. This molecular mechanism is common to all polyQ diseases and key networks are likely shared between the diseases.

Friedreich Ataxia Friedreich ataxia (FRDA) serves as an exciting example of the rapid progress made in therapeutics. In FRDA, the GAA.TTC triplet repeat sequence results in transcriptional silencing of the frataxin gene [20]. Frataxin is a mitochondrial protein and is a component of a multiprotein complex that assembles iron-sulfur clusters. Gottesfeld reviews the molecular mechanisms of FRDA and the numerous interesting approaches to treat the disease [21]. These include excision or correction of the frataxin gene by genome engineering, gene activation with small molecules, protein replacement, and small molecules identified in cellular screens. The therapeutic approaches for FRDA set the stage for many of the repeat expansion disorders.

SCA2 In 1996, the CAG expansion in ataxin-2 gene was discovered to cause Spinocerebellar ataxia type 2 (SCA2) [22-24]. Egorova and Bezprozvanny describe the epidemiology, the function of ataxin-2 in RNA metabolism and stress granules, the molecular changes underlying Purkinje cell loss, the cerebellar-thalamic cortical circuit dysregulation and ataxia, and molecular mechanisms that may be therapeutically targeted [25].

SCA7 Spinocerebellar ataxia type 7 (SCA7) is caused by a CAG expansion in the ataxin-7 gene and is characterized by neuronal loss in the cerebellum, brainstem, and retina. The major symptoms are cerebellar ataxia and blindness. The ataxin-7 protein is a subunit of the multiprotein SAGA complex which is involved in chromatin remodeling and there is now a detailed molecular understanding of how the polyQ expansion in ataxin-7 disrupts neuronal function. Niewiadomska-Cimicka and Trottier describe the molecular functions of ataxin-7 and detail current approaches to treat the disease [26]. 
SCA17 SCA17 is caused by a CAG/CAA repeat expansion in the gene encoding the TATA box-binding protein (TBP) [27]. TBP is a well-characterized transcription factor. Liu et al. discuss the normal function TBP, the transcriptional alterations caused by the polyQ expansion in TBP and thoughtfully review the current candidate drugs and reducing the expression of the disease genes by antisense oligonucleotides or CRISPR-Cas9 for the treatment of SCA17 [28].

SCA31 SCA31 is an adult onset neurological disorder with progression cerebellar ataxia caused by degenerating Purkinje cell in the Japanese population. The genetics of SCA31 was elucidated in 2009 [29]. There is $2.5-3.8 \mathrm{~kb}$ insertion of the penta-nucleotide repeat (TGGAA)n stretch in the introns of thymidine kinase 2 (TK) and BEAN (brain expressed, associated with Nedd4). As described by Drs. Ishikawa and Nagai, an emerging mechanism of RNA toxicity is apparent for this disease [30].

C9orf72 ALS/FTD In 2011, a hexanucleotide repeat expansion in the first intron of the C9orf72 gene was identified as a case of amyotrophic lateral sclerosis (ALS) and frontotemporal dementia (FTD). Given its relatively recent discovery, there has been rapid progress in identifying key pathogenic events such as the gain of toxicity from bidirectional transcribed repeat-containing RNAs, nucleocytoplasmic transport defects common to a number of the expansion diseases, and how the expansion causes two different diseases. These advances have led to phase I clinical trials using antisense oligonucleotide therapies. Drs. Jiang and Ravits review the exciting developments in our understanding of the pathogenic mechanisms for C9orf72 ALS/FTD [31].

Summary and Outlook It has been over three decades since the discovery of the first repeat expansion disease and the scientific community has provided a rich body of research that has elucidated the mechanisms that drive disease progression. All the reviews highlight how our understanding of the normal function of the disease-causing protein has evolved and the disruption of the function or gain of function provides valuable information for the development of therapies for these diseases. The discovery of new forms of repeat expansion diseases continues to fuel the field (Fig. 1). X-linked dystonia-parkinsonism (XDP) is associated with a SINE-VNTR-Alu (SVA)-type retrotransposon insertion in the TAF1 gene (TATA-box binding protein associated factor 1) which has a hexameric DNA repeat expansion, (CCCTCT)n, the length of which varies among patients and is inversely correlated to age of disease onset [32]. For many of the repeat expansion diseases, the use of antisense oligonucleotides or CRISPR-Cas9 for the treatment of these diseases offers a promising area of neurotherapeutics.

Required Author Forms Disclosure forms provided by the authors are available with the online version of this article.

\section{References}

1. Ellerby LM, Andrusiak RL, Wellington CL, Hackam AS, Propp SS, Wood JD, et al. Cleavage of atrophin-1 at caspase site aspartic acid 109 modulates cytotoxicity. J Biol Chem. 1999;274(13):8730 6.

2. Ellerby LM, Hackam AS, Propp SS, Ellerby HM, Rabizadeh S, Cashman NR, et al. Kennedy's disease: caspase cleavage of the androgen receptor is a crucial event in cytotoxicity. J Neurochem. 1999;72(1):185-95.

3. Gafni J, Hermel E, Young JE, Wellington CL, Hayden MR, Ellerby LM. Inhibition of calpain cleavage of huntingtin reduces toxicity: accumulation of calpain/caspase fragments in the nucleus. J Biol Chem. 2004;279(19):20211-20.

4. Gafni J, Papanikolaou T, Degiacomo F, Holcomb J, Chen S, Menalled L, et al. Caspase- 6 activity in a BACHD mouse modulates steady-state levels of mutant huntingtin protein but is not necessary for production of a 586 amino acid proteolytic fragment. J Neurosci. 2012;32(22):7454-65.

5. LaFevre-Bernt MA, Ellerby LM. Kennedy's disease. Phosphorylation of the polyglutamine-expanded form of androgen receptor regulates its cleavage by caspase- 3 and enhances cell death. J Biol Chem. 2003;278(37):34918-24.

6. Leyva MJ, Degiacomo F, Kaltenbach LS, Holcomb J, Zhang N, Gafni J, et al. Identification and evaluation of small molecule pancaspase inhibitors in Huntington's disease models. Chem Biol. 2010;17(11):1189-200.

7. Wellington CL, Ellerby LM, Gutekunst CA, Rogers D, Warby S, Graham RK, et al. Caspase cleavage of mutant huntingtin precedes neurodegeneration in Huntington's disease. J Neurosci. 2002;22(18):7862-72.

8. Wellington CL, Ellerby LM, Hackam AS, Margolis RL, Trifiro MA, Singaraja R, et al. Caspase cleavage of gene products associated with triplet expansion disorders generates truncated fragments containing the polyglutamine tract. J Biol Chem. 1998;273(15): 9158-67.

9. Wellington CL, Singaraja R, Ellerby L, Savill J, Roy S, Leavitt B, et al. Inhibiting caspase cleavage of huntingtin reduces toxicity and aggregate formation in neuronal and nonneuronal cells. J Biol Chem. 2000;275(26):19831-8.

10. La Spada AR, Wilson EM, Lubahn DB, Harding AE, Fischbeck $\mathrm{KH}$. Androgen receptor gene mutations in X-linked spinal and bulbar muscular atrophy. Nature. 1991;352(6330):77-9.

11. Arnold FJ, Merry DE. Molecular Mechanisms and Therapeutics for SBMA/Kennedy's Disease. Neurotherapeutics. 2019.

12. A novel gene containing a trinucleotide repeat that is expanded and unstable on Huntington's disease chromosomes. The Huntington's Disease Collaborative Research Group. Cell. 1993;72(6):971-83.

13. Maiuri T, Suart CE, Hung CLK, Graham KJ, Barba Bazan CA, Truant R. DNA Damage Repair in Huntington's Disease and Other Neurodegenerative Diseases. Neurotherapeutics. 2019.

14. Creus-Muncunill J, Ehrlich ME. Cell-Autonomous and Non-cellAutonomous Pathogenic Mechanisms in Huntington's Disease: Insights from In Vitro and In Vivo Models. Neurotherapeutics. 2019.

15. Naphade S, Tshilenge KT, Ellerby LM. Modeling Polyglutamine Expansion Diseases with Induced Pluripotent Stem Cells. Neurotherapeutics. 2019.

16. Orr HT, Chung MY, Banfi S, Kwiatkowski TJ, Jr., Servadio A, Beaudet AL, et al. Expansion of an unstable trinucleotide CAG repeat in spinocerebellar ataxia type 1. Nat Genet. 1993;4(3):221-6.

17. Srinivasan SR, Shakkottai VG. Moving Towards Therapy in SCA1: Insights from Molecular Mechanisms, Identification of Novel Targets, and Planning for Human Trials. Neurotherapeutics. 2019. 
18. Takiyama Y, Nishizawa M, Tanaka H, Kawashima S, Sakamoto H, Karube Y, et al. The gene for Machado-Joseph disease maps to human chromosome 14q. Nat Genet. 1993;4(3):300-4.

19. Da Silva JD, Teixeira-Castro A, Maciel P. From Pathogenesis to Novel Therapeutics for Spinocerebellar Ataxia Type 3: Evading Potholes on the Way to Translation. Neurotherapeutics. 2019.

20. Campuzano V, Montermini L, Molto MD, Pianese L, Cossee M, Cavalcanti F, et al. Friedreich's ataxia: autosomal recessive disease caused by an intronic GAA triplet repeat expansion. Science. 1996;271(5254):1423-7.

21. Gottesfeld JM. Molecular Mechanisms and Therapeutics for the GAA.TTC Expansion Disease Friedreich Ataxia. Neurotherapeutics. 2019.

22. Imbert G, Saudou F, Yvert G, Devys D, Trottier Y, Garnier JM, et al. Cloning of the gene for spinocerebellar ataxia 2 reveals a locus with high sensitivity to expanded CAG/glutamine repeats. Nat Genet. 1996;14(3):285-91.

23. Pulst SM, Nechiporuk A, Nechiporuk T, Gispert S, Chen XN, Lopes-Cendes I, et al. Moderate expansion of a normally biallelic trinucleotide repeat in spinocerebellar ataxia type 2. Nat Genet. 1996;14(3):269-76.

24. Sanpei K, Takano H, Igarashi S, Sato T, Oyake M, Sasaki H, et al. Identification of the spinocerebellar ataxia type 2 gene using a direct identification of repeat expansion and cloning technique, DIRECT. Nat Genet. 1996;14(3):277-84.

25. Egorova PA, Bezprozvanny IB. Molecular Mechanisms and Therapeutics for Spinocerebellar Ataxia Type 2. Neurotherapeutics. 2019.
26. Niewiadomska-Cimicka A, Trottier Y. Molecular Targets and Therapeutic Strategies in Spinocerebellar Ataxia Type 7. Neurotherapeutics. 2019.

27. Koide R, Kobayashi S, Shimohata T, Ikeuchi T, Maruyama M, Saito M, et al. A neurological disease caused by an expanded CAG trinucleotide repeat in the TATA-binding protein gene: a new polyglutamine disease? Hum Mol Genet. 1999;8(11):204753.

28. Liu Q, Pan Y, Li XJ, Li S. Molecular Mechanisms and Therapeutics for SCA17. Neurotherapeutics. 2019.

29. Sato N, Amino T, Kobayashi K, Asakawa S, Ishiguro T, Tsunemi T, et al. Spinocerebellar ataxia type 31 is associated with "inserted" penta-nucleotide repeats containing (TGGAA)n. Am J Hum Genet. 2009;85(5):544-57.

30. Ishikawa K, Nagai Y. Molecular Mechanisms and Future Therapeutics for Spinocerebellar Ataxia Type 31 (SCA31). Neurotherapeutics. 2019.

31. Jiang J, Ravits J. Pathogenic Mechanisms and Therapy Development for C9orf72 Amyotrophic Lateral Sclerosis/ Frontotemporal Dementia. Neurotherapeutics. 2019.

32. Bragg DC, Sharma N, Ozelius LJ. X-Linked DystoniaParkinsonism: recent advances. Curr Opin Neurol. 2019;32(4): 604-9.

Publisher's Note Springer Nature remains neutral with regard to jurisdictional claims in published maps and institutional affiliations. 